\title{
THE ELECTRON BEAM REACTION OF BENZOPHENONE TYPE POLYIMIDE
}

\author{
TAKAFUMI KUDO, TAKASHI YAMASHITA ${ }^{\dagger}$ and KAZUYUKI HORIE \\ Department of Reaction chemistry, Faculty of Engineering, the University of Tokyo, 7-3-1 Hongo, \\ Bunkyo-ku, Tokyo 113, JAPAN \\ $\dagger$ Research Center for Advanced Science and Technology, the University of Tokyo, 4-6-1 Komaba, \\ Meguro-ku, Tokyo 153, JAPAN
}

\section{Introduction}

Recently, photosensitive polyimides have been attracting much interest, because they can endure the high temperature and shorten the manufacturing process of integrated circuits. We have investigated the photoreaction of PI(BTDA/DEDPM), one of the negative type photosensitive polyimide, and clarified that the photocrosslinking occurs by intermolecular coupling of radicals, which are produced via the triplet excited state of benzophenone moiety [1]. Photosensitive polyimides are expected to be applied to the electron beam lithography, because the electron beam irradiation is thought to produce a higher exited state of the molecule. But few examples of the electron beam lithography of polyimide are known [2], so in this study we examined an application of PI(BTDA/DEDPM) to the electron beam lithography and investigated the characteristics and the mechanism of the reaction.

\section{Experimental}

The polyamic acid, PAA(BTDA/DEDPM), was prepared from benzophenonetetracarboxylic dianhydride (BTDA) and 3,3'-dimethyl-4,4'-diaminodiphenylmethane (DEDPM) in DMF solution. The resulting PAA was imidized with acetic anhydride and pyridine at room temperature. 
The sample films were prepared from a dichloroethane solution with a spin-coater onto 2.5 -inch silicon wafers. The electron beam (EB) irradiation was carried out with a Hitachi S-2100 type scanning electron microscope, of which acceleration voltage was $25 \mathrm{kV}$. The irradiation dose range from $10^{-7} \mathrm{C} / \mathrm{cm}^{2}$ to $10^{-3} \mathrm{C} / \mathrm{cm}^{2}$. After the electron beam irradiation the samples were developed with $\mathrm{CH}_{2} \mathrm{Cl}_{2}$ or with a mixed solvent of $\mathrm{CH}_{2} \mathrm{Cl}_{2}$ :iso-propyl alcohol (5:2), and the patterns were monitored with the electron microscope. The thickness of the films were measured with a Veeco Dektak-IIA type surface profile measuring system.

\section{Results and discussion}

Figure 1 shows a developed pattern of PI(BTDA/DEDPM) after $1.5 \times 10^{-3} \mathrm{C} / \mathrm{cm}^{2}$ electron beam irradiation. This photograph shows a negative pattern, which is similar with the case of photo irradiation. The IR spectra of the sample films before and after EB irradiation were measured, but no remarkable change was observed. These results suggest that the EB irradiation causes the crosslinking of the polymer, and the pattern is formed by the gelation. The Dose curve for PI(BTDA/DEDPM), whose molecular weight is $8 \times 10^{4}$ with $\mathrm{CH}_{2} \mathrm{Cl}_{2}$ as a developer, is shown in Figure 2. The first pattern was observed after $1 \times 10^{-5} \mathrm{C} / \mathrm{cm}^{2} \mathrm{~EB}$ irradiation, where the gelation is thought to begin. The sensitivity is determined to be $9 \times 10^{-5} \mathrm{C} / \mathrm{cm}^{2}$ and the contrast to be 0.5 from the curve. The dose curve for the sample whose molecular weight is $3.3 \times 10^{4}$ and which was developed with a mixed solvent of $\mathrm{CH}_{2} \mathrm{Cl}_{2}$ : iso-propyl alcohol $(5: 2)$ is shown in Figure 3 . The gelation began at $4 \times 10^{-5} \mathrm{C} / \mathrm{cm}^{2} \mathrm{~EB}$ irradiation due to the smaller initial molecular weight. The sensitivity is determined to be $9 \times 10^{-5}{\mathrm{C} / \mathrm{cm}^{2}}^{2}$ and its contrast is 1.4 , which is mainly due to the change in the developer.

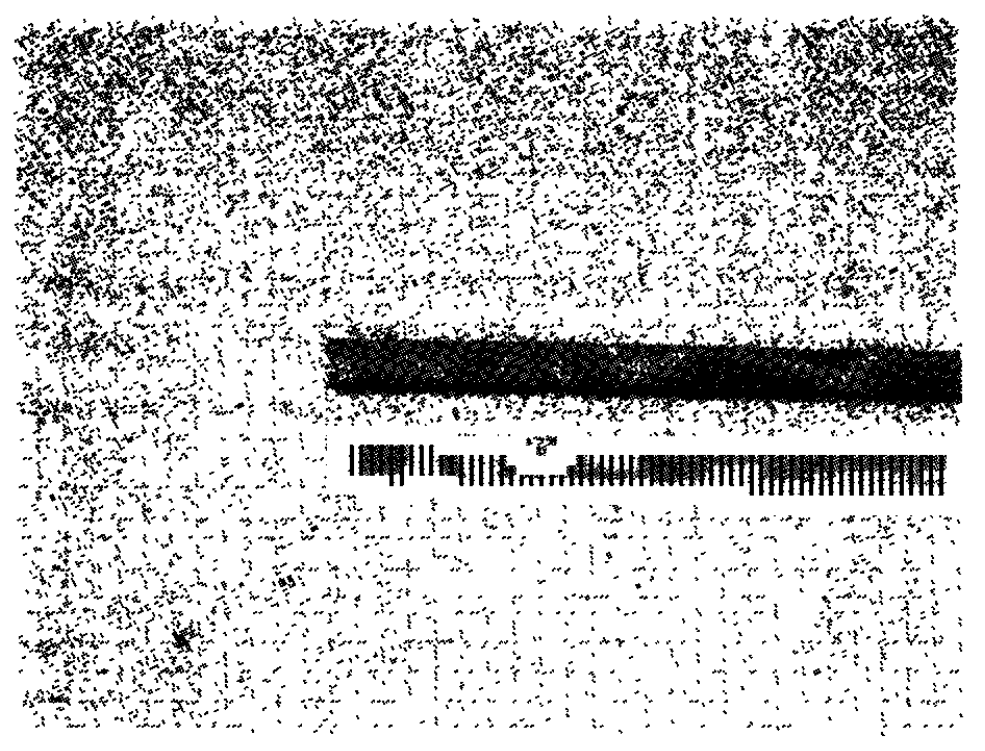

Figure 1. Micrograph of patterns of PI(BTDA/DEDPM) developed with a mixed solvent of $\mathrm{CH}_{2} \mathrm{Cl}_{2}$ and iso-propyl alcohl (5:2) after $1.5 \times 10^{-3} \mathrm{C} / \mathrm{cm}^{2} \mathrm{~EB}$ irradiation. 


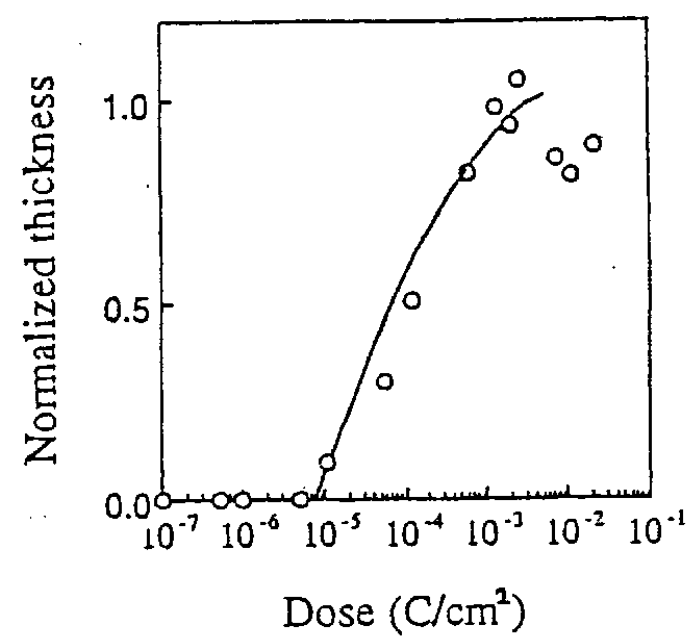

Figure 2. Change in the normalized thickness for PI(BTDA/DEDPM) $\left(M_{W}=8 \times 10^{4}\right)$ developed with $\mathrm{CH}_{2} \mathrm{Cl}_{2}$ during $\mathrm{EB}$ irradiation.

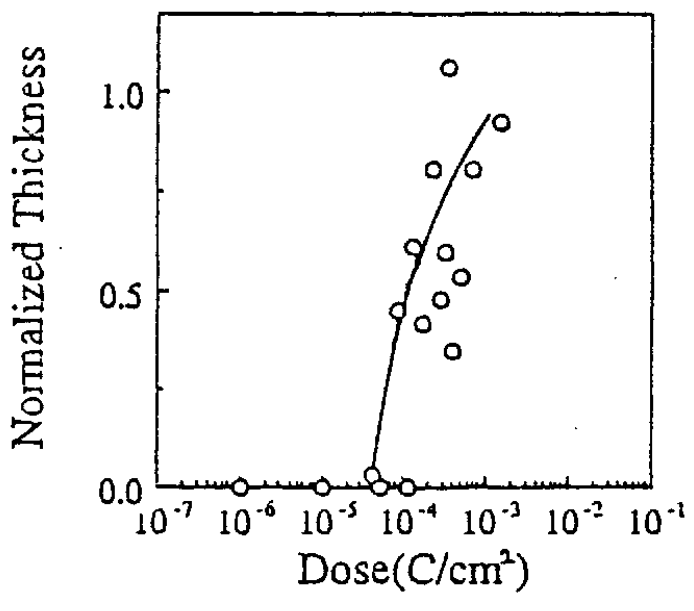

Figure 3. Change in the normalized thickness for $\mathrm{PI}(\mathrm{BTDA} / \mathrm{DEDPM})\left(\mathrm{M}_{\mathrm{w}}=3 \times 10^{4}\right)$ developed with $\mathrm{CH}_{2} \mathrm{Cl}_{2}$ and iso-propyl alcohol $(5: 2)$ during $\mathrm{EB}$ irradiation.

The sensitivity for the EB resist, OEBR 1000, which contains polymethyl methacrylate (PMMA) is $5 \times 10^{-5} \mathrm{C} / \mathrm{cm}^{2}$, that is almost comparable to that of PI(BTDA/DEDPM). In order to determine the mechanism of the reaction, the changes in the molecular weight distribution of PI(BTDA/DEDPM) during electron beam irradiation were measured by the gel permeation chromatography (GPC), which are shown in Figure 4.

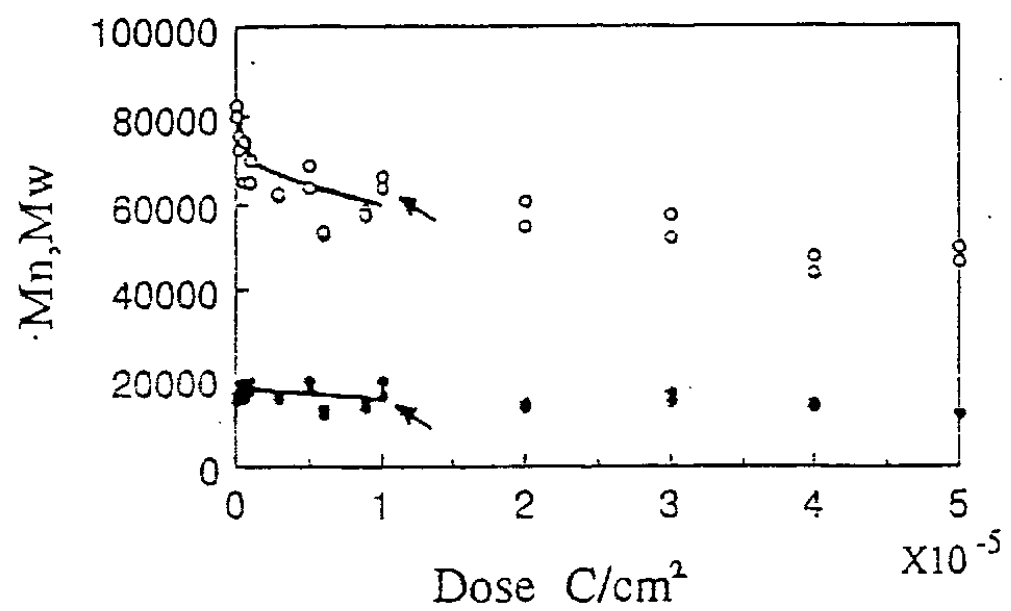

Figure 4. The criange in number and weight average molecular weights of PI(BTDAVDEDPM) during EB irradiation. Arrows show the critical dose for gelation. The values for doses above the critical point correspond to sol parts of the irradiated samples. 
The weight-average molecular weight decreases before gelation with the increase in electron beam intensity, while the number-average molecular weight decreases not so much. This shows that the main chain scission and crosslinking occur simultaneously, considering that PI(BTDA/DEDPM) gives the negative pattern by the electron beam irradiation. The geled sample after $10^{-4} \mathrm{C} / \mathrm{cm}^{2} \mathrm{~EB}$ irradiation could not be dissolved also in other good solvents such as NMP and DMF, showing that the highly irradiated sample is chemically crosslinked.

\section{Conclusion}

The EB lithography was performed with a photosensitive polyimide PI(BTDA/DEDPM). The

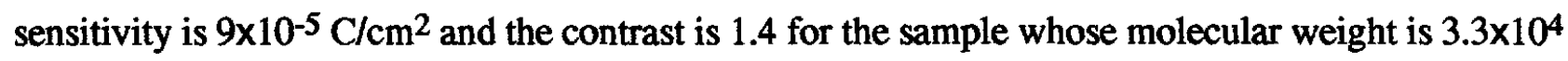
after development with a mixed solvent. The glass transition temperature $\left(T_{g}\right)$ for PI(BTDA/DEDPM) is $225^{\circ} \mathrm{C}$, which is great advantage to the PMMA containing EB resist, whose $\mathrm{T}_{\mathrm{g}}$ is about $100^{\circ} \mathrm{C}$. The increase in the sensitivity is expected by reducing the main chain scission of PI(BTDA/DEDPM) during EB irradiation.

\section{Reference}

1. H. Higuchi, T. Yamashita, K. Horie, I. Mita, Chem. Mater, 3, 188 (1991).

2.James.C, W.Chien, B.M. Gong, Polym.Eng.Sci, 14, 29, (1989) 